\title{
SEMI-GLOBAL ROBUST OUTPUT REGULATION FOR NONLINEAR SYSTEMS IN NORMAL FORM USING OUTPUT FEEDBACK*
}

\author{
WEIYAO LAN $^{\dagger}$, ZHIYONG CHEN ${ }^{\ddagger}$, AND JIE HUANG ${ }^{\S}$
}

\begin{abstract}
This paper studies the semi-global robust output regulation problem for the class of nonlinear affine systems in normal form. The same problem was studied before by Khalil under the assumption that the solution of the regulator equations is polynomial. By using the nonlinear internal model approach, we have relaxed the polynomial assumption on the solution of the regulator equations.
\end{abstract}

Key word: Output regulation; stabilization; output feedback; robust control; internal model.

1. Introduction. The nonlinear output regulation problem has been extensively studied since 1990s in [5], [6], and [8]. The robust version of this problem is addressed in [1], [2], and [4]. Also, the solvability of the various versions of the regional and/or semi-global robust output regulation problem are studied in [7], [9], [11], [12], and [14]. Nevertheless, the results of all these papers rely on a key assumption that the regulator equations of the system admit a polynomial solution. This assumption essentially requires that the nonlinear systems contain only polynomial nonlinearities. Recently, a general framework for studying the robust output regulation problem is proposed in [3]. Under this framework, the robust output regulation problem for a given plant can be systematically converted into a robust stabilization problem for an appropriately defined augmented system. Moreover, this framework admits a class of nonlinear internal models such that the polynomial condition (see, e.g., [7] and [12]) can be relaxed by some milder assumption. In this paper, we will further apply this framework to study a semi-global robust output regulation problem for a class of nonlinear systems in normal form. As in [3], our approach consists of two steps. First, convert the semi-global robust output regulation problem of the given system into a semi-global robust stabilization problem of an appropriately defined augmented

*The second author was partially supported by the National Natural Science Foundation of China under Grant 60504009. The third author was partially supported by the Hong Kong Research Grant Council under Grant CUHK4196/04E, and the National Natural Science Foundation of China under Grant 60374038

$\dagger$ Department of Electrical and Computer Engineering, National University of Singapore, Singapore, 117576, E-mail: elelw@nus.edu.sg

$\ddagger$ College of Automation Science and Engineering, South China University of Technology, Guang Zhou 510641, China, E-mail: auzychen@scut.edu.cn

$\S$ Department of Automation and Computer-Aided Engineering, Chinese University of Hong Kong, Shatin, NT, Hong Kong, Tel: +00852-26098473, Fax: +00852-26036002, E-mail: jhuang@acae.cuhk.edu.hk 
system. Second, solve the semi-global robust stabilization problem of the augmented system using the approach adapted from the work of [13] and [14]. It should be noted that while the first step follows straightforwardly from the framework developed in [3], the accomplishment of the second step is non-trivial. This is because the zero dynamics of the augmented system consist of two parts. The first part is a variation of the zero dynamics of the given plant, and the second part is the dynamics that govern the nonlinear internal model. We have employed the changing supply function technique [16] to address the issue of the stability property of the zero dynamics of the augmented system.

The paper is organized as follows. Section 2 formulates a semi-global robust output regulation problem, and converts this semi-global robust output regulation problem into a semi-global robust stabilization problem for an augmented system. In Section 3 , we establish the solvability conditions for the semi-global robust stabilization problem of the augmented system by dynamic output feedback, which in turn leads to the solvability conditions for the semi-global output regulation problem of the original system. Section 4 gives an example to illustrate our method. Finally, Section 5 concludes this paper with some remarks.

2. Problem Description and Problem Conversion. Consider a nonlinear system described as follows,

$$
\begin{aligned}
\dot{z} & =f_{0}(z, x, v, w) \\
\dot{x}_{i} & =x_{i+1}, i=1, \cdots, r-1 \\
\dot{x}_{r} & =f_{1}(z, x, v, w)+u \\
\dot{v} & =A_{1} v \\
e & =x_{1}-q(v, w)
\end{aligned}
$$

where $z \in \mathbb{R}^{m}, x=\operatorname{col}\left(x_{1}, \cdots, x_{r}\right)$ with $x_{i} \in \mathbb{R}, i=1, \cdots, r$, are the plant states, $u \in \mathbb{R}$ is the control input, $e \in \mathbb{R}$ is the tracking error, $v \in \mathbb{R}^{q}$ is the exogenous signal representing the disturbance and/or the reference input, and $w \in \mathbb{R}^{N}$ is the uncertain parameter. All the functions are sufficiently smooth with $f_{0}(0,0,0, w)=0$, $f_{1}(0,0,0, w)=0$, and $q(0, w)=0$. The exosystem is neutrally stable, i.e., all the eigenvalues of $A_{1}$ are simple and have zero real parts.

The class of output feedback control laws considered here are described by

$$
u=k_{c}\left(z_{c}, e\right), \quad \dot{z}_{c}=f_{c}\left(z_{c}, e\right)
$$

where $z_{c}$ is the compensator state vector of dimension $n_{c}$ to be specified later, and $k_{c}$ and $f_{c}$ are globally defined sufficiently smooth functions.

Semi-global robust output regulation problem. Given compact sets $\mathbb{V}_{0} \subset \mathbb{R}^{q}$, $\mathbb{W} \subset \mathbb{R}^{N}, \mathbb{Z} \subset \mathbb{R}^{m}, \mathbb{X} \subset \mathbb{R}^{r}$ and $\mathbb{Z}_{c} \subset \mathbb{R}^{n_{c}}$, which contain the origins of the respective 
Euclidean spaces, a controller of the form (2) is said to solve the robust output regulation problem for the system (1) with respect to $\mathbb{V}_{0} \times \mathbb{W} \times \mathbb{Z} \times \mathbb{X} \times \mathbb{Z}_{c}$ if the closed-loop system (1)-(2) with its state being denoted by $x_{c}=\operatorname{col}\left(z, x, z_{c}\right)$ has the following two properties:

Property 1. For any $v(0) \in \mathbb{V}_{0}, w \in \mathbb{W}$, and $x_{c}(0) \in \mathbb{X}_{c}$ where $\mathbb{X}_{c}=$ $\mathbb{Z} \times \mathbb{X} \times \mathbb{Z}_{c}$, the trajectories of the closed-loop system starting from $x_{c}(0)$ and $v(0)$ exist and are bounded for all $t \geq 0$.

Property 2. The tracking error $e(t)$ of the trajectories described in Property 1 approaches zero asymptotically, i.e., $\lim _{t \rightarrow \infty} e(t)=0$.

If for any given triple $\left(\mathbb{V}_{0}, \mathbb{W}, \mathbb{X}_{c}\right)$, there exists a controller of the form (2) such that the closed-loop system (1)-(2) has Properties 1 and 2, then we say that the semi-global robust output regulation problem of the system (1) is solvable.

REMARK 2.1. Since the exosystem is neutrally stable, for any $v(0) \in \mathbb{V}_{0}$, there exists a compact set $\mathbb{V} \subset \mathbb{R}^{q}$ containing the origin of $\mathbb{R}^{q}$ such that $v(t) \in \mathbb{V}$ for all $t \geq 0$.

Let us first apply the framework developed in [3] to convert the semi-global robust output regulation problem into a semi-global robust stabilization problem for an appropriately defined augmented system. To fulfill this conversion, we need the following standard assumptions.

A1: Let $\mathbf{x}(v, w)=\operatorname{col}\left(\mathbf{x}_{1}(v, w), \cdots, \mathbf{x}_{r}(v, w)\right)$ where

$$
\begin{aligned}
& \mathbf{x}_{1}(v, w)=q(v, w) \\
& \mathbf{x}_{i}(v, w)=\frac{\partial \mathbf{x}_{i-1}(v, w)}{\partial v} A_{1} v, \quad i=2, \cdots, r .
\end{aligned}
$$

There exists a sufficiently smooth function $\mathbf{z}(v, w)$ with $\mathbf{z}(0,0)=0$ such that, for all $v \in \mathbb{R}^{q}, w \in \mathbb{R}^{N}$,

$$
\frac{\partial \mathbf{z}(v, w)}{\partial v} A_{1} v=f_{0}(\mathbf{z}(v, w), \mathbf{x}(v, w), v, w) .
$$

REMARK 2.2. Under assumption A1, the regulator equations associated with the system (1) are solvable by $(\mathbf{z}(v, w), \mathbf{x}(v, w), \mathbf{u}(v, w))$ where

$$
\mathbf{u}(v, w)=\frac{\partial \mathbf{x}_{r}(v, w)}{\partial v} A_{1} v-f_{1}(\mathbf{z}(v, w), \mathbf{x}(v, w), v, w) .
$$

However, the solvability of the regulator equations is insufficient for solving the robust output regulation problem, some additional conditions have to be imposed on the solution of the regulator equations (see [1], [2], [4], [7], and [12]). Despite the different appearances of these conditions, they amount to requiring the system admit a linear internal model, which in turn essentially requires the system only contain polynomial nonlinearities. Recently, a much less restrictive condition was given in [3] and will be stated below as assumption A2. 
A2: There exist an integer $s$ and sufficiently smooth functions $\tau: \mathbb{R}^{q+N} \mapsto \mathbb{R}^{s}$ and $\Gamma: \mathbb{R}^{s} \mapsto \mathbb{R}$ vanishing at the origin and a matrix $\Phi \in \mathbb{R}^{s \times s}$ such that, for all trajectories $v(t)$ of the exosystem and all $w \in \mathbb{R}^{N}$,

$$
\frac{d \tau(v, w)}{d t}=\Phi \tau(v, w), \quad \mathbf{u}(v, w)=\Gamma(\tau(v, w)) .
$$

Moreover, let $\Psi$ be the gradient of $\Gamma(\tau)$ at the origin. Then the pair $(\Psi, \Phi)$ is observable.

REMARK 2.3. For any nonsingular matrix $T$ of dimension $s$, let $\theta=T \tau$, $\alpha(\theta)=T \Phi T^{-1} \theta$, and $\beta(\theta)=\Gamma\left(T^{-1} \theta\right)$. Then, the triplet $\{\theta, \alpha, \beta\}$ is what is called the steady-state generator of system (1) with output $u$ [3]. It is noted when the solution of the regulator equations is polynomial, assumption A2 is satisfied automatically with $\Gamma$ a linear function of $\tau$. For this special case, the semi-global stabilization of systems (1) has been studied in [9] and [12]. What is interesting here is that assumption A2 can be satisfied with $\Gamma$ a nonlinear function of $\tau$ when the solution of the regulator equations is not polynomial [3].

REMARK 2.4. Under assumptions A1-A2, since the pair $(\Psi, \Phi)$ is observable, for any controllable pair $(M, N)$ with $M$ a Hurwitz matrix of dimension $s$ and $N$ a column vector, there exists a nonsingular matrix $T$ satisfying the Sylvester equation $T \Phi-M T=N \Psi$. Define

$$
\dot{\eta}=\gamma(\eta, u)=M \eta+N\left(u-\beta^{[2]}(\eta)\right)
$$

where $\eta \in \mathbb{R}^{s}$ and $\beta^{[2]}(\eta)=\beta(\eta)-\Psi T^{-1} \eta$. System (4) is introduced in [3] and is called an internal model of (1) with output $u$. It is noted that when the function $\beta(\cdot)$ is linear, (4) reduces to a linear internal model.

The combination of the system (1) and the internal model (4) is called the augmented system. Under the coordinate and input transformation,

$$
\begin{aligned}
\bar{\eta} & =\eta-\theta(v, w) \\
\bar{z} & =z-\mathbf{z}(v, w) \\
\bar{x}_{i} & =x_{i}-\mathbf{x}_{i}(v, w), \quad i=1, \cdots, r \\
\bar{u} & =u-\beta(\eta)
\end{aligned}
$$

the augmented system takes the following form

$$
\begin{aligned}
\dot{\bar{\eta}} & =\left(M+N \Psi T^{-1}\right) \bar{\eta}+N \bar{u} \\
\dot{\bar{z}} & =\bar{f}_{0}\left(\bar{z}, \bar{x}_{1}, \cdots, \bar{x}_{r}, v, w\right) \\
\dot{\bar{x}}_{i} & =\bar{x}_{i+1}, i=1, \cdots, r-1 \\
\dot{\bar{x}}_{r} & =\bar{f}_{1}\left(\bar{\eta}, \bar{z}, \bar{x}_{1}, \cdots, \bar{x}_{r}, v, w\right)+\bar{u} \\
\dot{v} & =A_{1} v \\
e & =\bar{x}_{1}
\end{aligned}
$$


where

$$
\begin{aligned}
& \bar{f}_{0}\left(\bar{z}, \bar{x}_{1}, \cdots, \bar{x}_{r}, v, w\right) \\
= & f_{0}\left(\bar{z}+\mathbf{z}(v, w), \bar{x}_{1}+\mathbf{x}_{1}(v, w), \cdots, \bar{x}_{r}+\mathbf{x}_{r}(v, w), v, w\right) \\
& -f_{0}\left(\mathbf{z}(v, w), \mathbf{x}_{1}(v, w), \cdots, \mathbf{x}_{r}(v, w), v, w\right) \\
& \bar{f}_{1}\left(\bar{\eta}, \bar{z}, \bar{x}_{1}, \cdots, \bar{x}_{r}, v, w\right) \\
= & f_{1}\left(\bar{z}+\mathbf{z}(v, w), \bar{x}_{1}+\mathbf{x}_{1}(v, w), \cdots, \bar{x}_{r}+\mathbf{x}_{r}(v, w), v, w\right) \\
& -f_{1}\left(\mathbf{z}(v, w), \mathbf{x}_{1}(v, w), \cdots, \mathbf{x}_{r}(v, w), v, w\right) \\
& +\beta(\bar{\eta}+\theta(v, w))-\beta(\theta(v, w)) .
\end{aligned}
$$

It is clear that

$$
\bar{f}_{0}(0, \cdots, 0, v, w)=0, \quad \bar{f}_{1}(0, \cdots, 0, v, w)=0
$$

that is, the origin $\operatorname{col}(\bar{\eta}, \bar{z}, \bar{x})=0$ is the equilibrium point of the unforced augmented system (6) for all trajectories $v(t)$ of the exosystem and any $w \in \mathbb{R}^{N}$.

Let $d(t)=\operatorname{col}(v(t), w) \in \mathbb{D}=\mathbb{V} \times \mathbb{W}$, then system (6) is a special case of the following more general nonlinear systems

$$
\dot{x}=f(x, u, d(t)), \quad e=h(x, d(t))
$$

where $x$ is the $n$-dimensional system state, $u$ the $m$-dimensional control input, $e$ the $p$ dimensional output, $d(t)$ the $n_{d}$-dimensional uncertain parameters, and $f(0,0, d(t))=$ 0 and $h(0, d(t))=0$ for all $d(t) \in \mathbb{D}$.

Semi-global robust stabilization problem. Consider the uncertain nonlinear control system (8) and a dynamic output feedback controller of the form

$$
u=k(\xi, e), \quad \dot{\xi}=\varphi(\xi, e)
$$

where $\xi \in \mathbb{R}^{n_{\xi}}$ for some integer $n_{\xi}$, and $k$ and $\varphi$ are globally defined sufficiently smooth functions satisfying $k(0,0)=0$ and $\varphi(0,0)=0$. Let $\mathbb{D} \subset \mathbb{R}^{n_{d}}, \mathbb{X} \subset \mathbb{R}^{n}$, and $\mathbb{S} \subset \mathbb{R}^{n_{\xi}}$ be some given compact sets containing the origins of the respective Euclidian spaces. If there exists a dynamic output feedback controller of the form (9) such that, for any $x(0) \in \mathbb{X}, \xi(0) \in \mathbb{S}$ and $d(t) \in \mathbb{D}$, the solution of the closed-loop system (8) and (9) exists and is bounded for all $t \geq 0$ and all the states converge to the origin asymptotically, then we say that the controller (9) solves the robust stabilization problem of the system (8) with respect to $\mathbb{D} \times \mathbb{X} \times \mathbb{S}$. If for every triple $(\mathbb{D}, \mathbb{X}, \mathbb{S})$, there exists a dynamic output feedback controller of the form (9) that solves the robust stabilization problem of the system (8) with respect to $\mathbb{D} \times \mathbb{X} \times \mathbb{S}$, then we say that the semi-global stabilization problem of the system (8) is solvable by output feedback control. 
As for the global robust output regulation case studied in [3], the following proposition shows that the solvability of the semi-global robust stabilization problem of the augmented system (6) leads to that of the semi-global robust output regulation problem of the original system (1).

Proposition 2.1. Consider the augmented system (6) with the uncertainty $d(t)=\operatorname{col}(v(t), w) \in \mathbb{D}=\mathbb{V} \times \mathbb{W}$. If a dynamic output feedback controller

$$
\bar{u}=k(\xi, e), \quad \dot{\xi}=\varphi(\xi, e)
$$

solves the robust stabilization problem of (6) with respect to $\mathbb{D} \times(\overline{\mathbb{E}} \times \overline{\mathbb{Z}} \times \overline{\mathbb{X}}) \times \mathbb{S}$ where $\overline{\mathbb{E}} \times \overline{\mathbb{Z}} \times \overline{\mathbb{X}} \subset \mathbb{R}^{s} \times \mathbb{R}^{m} \times \mathbb{R}^{r}$ are compact sets containing the origin (in particular, $\bar{\eta}(0) \in \overline{\mathbb{E}}, \bar{z}(0) \in \overline{\mathbb{Z}}$, and $\bar{x}(0) \in \overline{\mathbb{X}})$, then the dynamic output feedback controller of the form

$$
\begin{aligned}
u & =\beta(\eta)+k(\xi, e) \\
\dot{\eta} & =M \eta+N\left(k(\xi, e)+\Psi T^{-1} \eta\right) \\
\dot{\xi} & =\varphi(\xi, e)
\end{aligned}
$$

solves the robust output regulation problem of the original system (1) with respect to $\mathbb{V}_{0} \times \mathbb{W} \times \mathbb{Z} \times \mathbb{X} \times \mathbb{Z}_{c}$ where $\mathbb{Z}_{c}=\mathbb{E} \times \mathbb{S}$ and

$$
\begin{aligned}
& \mathbb{E}=\left\{\eta \in \mathbb{R}^{s}: \eta=\bar{\eta}+\theta(v, w), \bar{\eta} \in \overline{\mathbb{E}}, \operatorname{col}(v, w) \in \mathbb{D}\right\} \\
& \mathbb{Z}=\left\{z \in \mathbb{R}^{m}: z=\bar{z}+\mathbf{z}(v, w), \bar{z} \in \overline{\mathbb{Z}}, \operatorname{col}(v, w) \in \mathbb{D}\right\} \\
& \mathbb{X}=\left\{x \in \mathbb{R}^{r}: x=\bar{x}+\mathbf{x}(v, w), \bar{x} \in \overline{\mathbb{X}}, \operatorname{col}(v, w) \in \mathbb{D}\right\} .
\end{aligned}
$$

Proof. For any given compact sets $\overline{\mathbb{E}}, \overline{\mathbb{Z}}, \overline{\mathbb{X}}$ and $\mathbb{S}$ containing the origins of the respective Euclidian spaces, assume the output feedback controller (10) solves the robust stabilization problem of the system (6) with respect to $\mathbb{D} \times \overline{\mathbb{E}} \times \overline{\mathbb{Z}} \times \overline{\mathbb{X}} \times \mathbb{S}$, that is, for any $\operatorname{col}(\bar{\eta}(0), \bar{z}(0), \bar{x}(0), \xi(0)) \in \overline{\mathbb{E}} \times \overline{\mathbb{Z}} \times \overline{\mathbb{X}} \times \mathbb{S}$ and $d(t) \in \mathbb{D}$, the states $\bar{\eta}$, $\bar{z}, \bar{x}, \xi$ of the closed-loop system (6) and (10) are bounded for all $t \geq 0$ and converge to the origin asymptotically. Now, consider the closed-loop system (1) and (11), and denote $x_{c}=\operatorname{col}(z, x, \eta, \xi)$. Since $x_{c}=\operatorname{col}(\bar{z}+\mathbf{z}(v, w), \bar{x}+\mathbf{x}(v, w), \bar{\eta}+\theta(v, w), \xi), x_{c}$ is also bounded for all $t \geq 0$ for any $x_{c}(0)=\operatorname{col}(z(0), x(0), \eta(0), \xi(0)) \in \mathbb{Z} \times \mathbb{X} \times \mathbb{E} \times \mathbb{S}$. Furthermore,

$$
\lim _{t \rightarrow \infty} e(t)=\lim _{t \rightarrow \infty}\left(x_{1}(t)-\mathbf{x}_{1}(v(t), w)\right)=\lim _{t \rightarrow \infty} \bar{x}_{1}(t)=0 .
$$

As a result, the controller (11) solves the robust output regulation problem of the original system (1) with respect to $\mathbb{V}_{0} \times \mathbb{W} \times \mathbb{Z} \times \mathbb{X} \times \mathbb{Z}_{c}$.

3. Robust Stabilization via Output Feedback. Due to Proposition 2.1, we only need to solve the semi-global robust stabilization problem of the augmented 
system (6) in order to solve the semi-global robust regulation problem of the original system (1). System (6) is in the standard normal form. The robust stabilization problem of the system of the form (6) has been well studied in literatures [13] and [14]. In particular, Khalil et al gave solvability conditions for the semi-global robust stabilization of the system of the form (6) when $d$ is constant [13]. To make use of the result of [13], let us introduce another transformation

$$
\tilde{\eta}=\bar{\eta}-N \bar{x}_{r}
$$

Then, the system (6) is transformed to

$$
\begin{aligned}
\dot{\tilde{\eta}} & =Q\left(\tilde{\eta}, \bar{z}, \bar{x}_{1}, \cdots, \bar{x}_{r}, v, w\right) \\
\dot{\bar{z}} & =\bar{f}_{0}\left(\bar{z}, \bar{x}_{1}, \cdots, \bar{x}_{r}, v, w\right) \\
\dot{\bar{x}}_{i} & =\bar{x}_{i+1}, i=1, \cdots, r-1 \\
\dot{\bar{x}}_{r} & =\bar{f}_{1}\left(\tilde{\eta}+N \bar{x}_{r}, \bar{z}, \bar{x}_{1}, \cdots, \bar{x}_{r}, v, w\right)+\bar{u} \\
e & =\bar{x}_{1}
\end{aligned}
$$

where

$$
\begin{aligned}
Q\left(\tilde{\eta}, \bar{z}, \bar{x}_{1}, \cdots, \bar{x}_{r}, v, w\right)= & M \tilde{\eta}+M N \bar{x}_{r}-N\left(\beta^{[2]}\left(\tilde{\eta}+N \bar{x}_{r}+\theta(v, w)\right)-\beta^{[2]}(\theta(v, w))\right) \\
& -N\left(f_{1}\left(\bar{z}+\mathbf{z}(v, w), \bar{x}_{1}+\mathbf{x}_{1}(v, w), \cdots, \bar{x}_{r}+\mathbf{x}_{r}(v, w), v, w\right)\right. \\
& \left.-f_{1}\left(\mathbf{z}(v, w), \mathbf{x}_{1}(v, w), \cdots, \mathbf{x}_{r}(v, w), v, w\right)\right) .
\end{aligned}
$$

It is clear that

$$
Q(0,0,0, \cdots, 0, v, w)=0 .
$$

Denote $d(t)=\operatorname{col}(v(t), w), \tilde{z}=\operatorname{col}(\tilde{\eta}, \bar{z})$ and $\bar{x}=\operatorname{col}\left(\bar{x}_{1}, \cdots, \bar{x}_{r}\right)$, then system (13) can be rewritten as

$$
\begin{aligned}
\dot{\tilde{z}} & =\phi_{0}(\tilde{z}, \bar{x}, d) \\
\dot{\bar{x}} & =A \bar{x}+B\left(\phi_{1}(\tilde{z}, \bar{x}, d)+\bar{u}\right) \\
e & =\bar{x}_{1}
\end{aligned}
$$

where

$$
A=\left[\begin{array}{cc}
0_{(r-1) \times 1} & I_{(r-1) \times(r-1)} \\
0 & 0_{1 \times(r-1)}
\end{array}\right], \quad B=\left[\begin{array}{c}
0_{(r-1) \times 1} \\
1
\end{array}\right]
$$

and

$$
\begin{aligned}
\phi_{0}(\tilde{z}, \bar{x}, d) & =\left[\begin{array}{c}
Q\left(\tilde{\eta}, \bar{z}, \bar{x}_{1}, \cdots, \bar{x}_{r}, v, w\right) \\
\bar{f}_{0}\left(\bar{z}, \bar{x}_{1}, \cdots, \bar{x}_{r}, v, w\right)
\end{array}\right] \\
\phi_{1}(\tilde{z}, \bar{x}, d) & =\bar{f}_{1}\left(\tilde{\eta}+N \bar{x}_{r}, \bar{z}, \bar{x}_{1}, \cdots, \bar{x}_{r}, v, w\right) .
\end{aligned}
$$

It is noted that $\phi_{0}(0,0, d)=0$ for all $d$.

It can be deduced from Corollary 1 of [13] that system (15) is semi-globally stabilizable if the system satisfies Conditions 1 to 3 listed below. 
Condition 1: There exist a $C^{1}$ function $W_{1}: \mathbb{R}^{m+s} \mapsto \mathbb{R}^{+}$, and class $K_{\infty}$ functions $\alpha_{i}, \mathrm{i}=1,2,3$, and $\gamma_{1}$ such that, for all $(\tilde{z}, \bar{x}) \in \mathbb{R}^{m+s} \times \mathbb{R}^{r}$, and all $d \in \mathbb{D}$,

$$
\begin{aligned}
& \alpha_{1}(\|\tilde{z}\|) \leq W_{1}(\tilde{z}) \leq \alpha_{2}(\|\tilde{z}\|) \\
& \frac{\partial W_{1}(\tilde{z})}{\partial \tilde{z}} \phi_{0}(\tilde{z}, \bar{x}, d) \leq-\alpha_{3}(\|\tilde{z}\|), \forall\|\tilde{z}\| \geq \gamma_{1}(\|\bar{x}\|) .
\end{aligned}
$$

Before introducing Condition 2, the following notations are defined. Let $K$ be such that $A+B K$ is Hurwitz, and the symmetric positive definite matrix $P$ be the solution of the Lyapunov equation

$$
P(A+B K)+(A+B K)^{T} P=-I .
$$

Also let

$$
\begin{aligned}
& \mathbb{O}_{c_{1}}^{1}=\left\{\tilde{z} \in \mathbb{R}^{m+s}: W_{1}(\tilde{z}) \leq c_{1}\right\} \\
& \mathbb{O}_{c_{2}}^{2}=\left\{\bar{x} \in \mathbb{R}^{r}: W_{2}(\bar{x}) \leq c_{2}\right\}
\end{aligned}
$$

where $W_{2}(\bar{x})=\bar{x}^{T} P \bar{x}$.

Condition 2: For each pair of $c_{1}, c_{2}>0$ satisfying

$$
\alpha_{4}\left(\sqrt{c_{2} / \lambda_{\min }(P)}\right) \leq c_{1}
$$

where $\lambda_{\min }(P)$ is the minimal eigenvalue of the matrix $P$, and $\alpha_{4}=\alpha_{2} \circ \gamma_{1}$, there exists a scalar nonnegative locally Lipschitz function $\rho(\bar{x})$ such that

$$
\left|\phi_{1}(\tilde{z}, \bar{x}, d)-K \bar{x}\right| \leq \rho(\bar{x})
$$

for all $(\tilde{z}, \bar{x}) \in \mathbb{O}_{c_{1}}^{1} \times \mathbb{O}_{c_{2}}^{2}$, all $d \in \mathbb{D}$.

Condition 3: In some neighborhood of $(\tilde{z}, \bar{x})=0$, there exist a $C^{1}$ positive definite function $\tilde{W}_{1}: \mathbb{R}^{m+s} \mapsto \mathbb{R}^{+}$, such that, for all $d \in \mathbb{D}$,

$$
\begin{aligned}
\frac{\partial \tilde{W}_{1}(\tilde{z})}{\partial \tilde{z}} \phi_{0}(\tilde{z}, 0, d) & \leq-\lambda_{1}\|\tilde{z}\|^{2} \\
\left|\phi_{1}(\tilde{z}, 0, d)\right| & \leq \lambda_{2}\|\tilde{z}\| \\
\frac{\partial \tilde{W}_{1}(\tilde{z})}{\partial \tilde{z}}\left[\phi_{0}(\tilde{z}, \bar{x}, d)-\phi_{0}(\tilde{z}, 0, d)\right] & \leq \lambda_{3}\|\tilde{z}\|\|\bar{x}\|
\end{aligned}
$$

where $\lambda_{i}>0, i=1,2,3$.

REMARK 3.1. Note that, in [13], the uncertainty $d$ is assumed to be constant. It is not difficult to conclude that the results of [13] also apply to the case where $d$ is time-varying as long as $d$ belongs to a compact set.

The zero dynamics of the augmented system (6) consist of the zero dynamics of the original plant and the dynamics governing the internal model. Therefore, even though 
the zero dynamics of the plant satisfy Conditions 1 to 3 , there is no guarantee that the zero dynamics of the augmented system also satisfy Conditions 1 to 3 . Therefore, it is interesting to further study the problem of imposing conditions on the plant and the internal model (4) so that the zero dynamics of the augmented system can satisfy Conditions 1 to 3 .

A3: There exist a $C^{1}$ function $V_{1}(\bar{z})$ and class $K_{\infty}$ functions $\bar{\alpha}_{i}, i=1,2,3$ and $\sigma$, independent on $d(t)$ such that, for all $(\bar{z}, \bar{x}) \in \mathbb{R}^{m} \times \mathbb{R}^{r}$, and all $d \in \mathbb{D}$,

$$
\begin{array}{r}
\bar{\alpha}_{1}(\|\bar{z}\|) \leq V_{1}(\bar{z}) \leq \bar{\alpha}_{2}(\|\bar{z}\|) \\
\frac{\partial V_{1}(\bar{z})}{\partial \bar{z}} \bar{f}_{0}(\bar{z}, \bar{x}, d) \leq-\bar{\alpha}_{3}(\|\bar{z}\|)+\sigma(\|\bar{x}\|),
\end{array}
$$

and in some neighborhood of $\bar{z}=0$,

$$
\left\|\frac{\partial V_{1}(\bar{z})}{\partial \bar{z}}\right\| \leq \bar{\lambda}_{1}\|\bar{z}\|, \lambda_{1}>0
$$

Moreover, the function $\bar{\alpha}_{3}(\cdot)$ satisfies

$$
\lim _{s \rightarrow 0^{+}} \sup \frac{\bar{\alpha}_{3}^{-1}\left(s^{2}\right)}{s}<+\infty .
$$

A4: Let the symmetric positive definite matrix $P_{M}$ be the solution of the Lyapunov equation

$$
P_{M} M+M^{T} P_{M}=-I .
$$

Then there exists a positive number $R$, such that, for all $\tilde{\eta}$ and $\delta$,

$$
-2 \tilde{\eta}^{T} P_{M} N\left(\beta^{[2]}(\tilde{\eta}+\delta)-\beta^{[2]}(\delta)\right) \leq(1-R) \tilde{\eta}^{T} \tilde{\eta} .
$$

TheOrem 3.1. Suppose the system (15) satisfies assumptions $A 3$ and $A 4$, then there exists a positive definite function $W_{1}(\tilde{z})$ such that $\alpha_{1}(\|\tilde{z}\|) \leq W_{1}(\tilde{z}) \leq \alpha_{2}(\|\tilde{z}\|)$ for some class $K_{\infty}$ functions $\alpha_{i}, i=1,2$, and

$$
\frac{\partial W_{1}(\tilde{z})}{\partial \tilde{z}} \phi_{0}(\tilde{z}, \bar{x}, d(t)) \leq-\alpha_{3}(\|\tilde{z}\|), \forall\|\tilde{z}\| \geq \gamma_{1}(\|\bar{x}\|)
$$

for some class $K_{\infty}$ functions $\gamma_{1}$ and $\alpha_{3}$. Further, suppose the system (15) satisfies the additional assumption

A5: For each pair of $c_{1}, c_{2}>0$ satisfying

$$
\alpha_{4}\left(\sqrt{c_{2} / \lambda_{\min }(P)}\right) \leq c_{1},
$$

where $\alpha_{4}=\alpha_{2} \circ \gamma_{1}$, there exists a scalar nonnegative locally Lipschitz function $\rho(\bar{x})$ such that

$$
\left|\phi_{1}(\tilde{z}, \bar{x}, d)-K \bar{x}\right| \leq \rho(\bar{x})
$$

for all $(\tilde{z}, \bar{x}) \in \mathbb{O}_{c_{1}}^{3} \times \mathbb{O}_{c_{2}}^{2}$, and all $d \in \mathbb{D}$ where $\mathbb{O}_{c_{1}}^{3}=\left\{\tilde{z} \in \mathbb{R}^{m+s}: V_{1}(\bar{z}) \leq\right.$ $\left.c_{1}, V_{2}(\tilde{\eta}) \leq c_{1}\right\}$ and $V_{2}(\tilde{\eta})=\tilde{\eta}^{T} P_{M} \tilde{\eta}$. 
Then, the semi-global robust stabilization problem of the system (15) is solvable by a dynamic output feedback controller of the form (9).

Proof. It suffices to prove that system (15) satisfies Condition 1 under assumptions $\mathrm{A} 3$ and A4, and Conditions 2 and 3 under assumptions A3-A5.

Verification of Condition 1: Consider the $\tilde{z}$-subsystem of (15), $\dot{\tilde{z}}=\phi_{0}(\tilde{z}, \bar{x}, d)$, that is,

$$
\begin{aligned}
& \dot{\tilde{\eta}}=Q(\tilde{\eta}, \bar{z}, \bar{x}, d(t)) \\
& \dot{\bar{z}}=\bar{f}_{0}(\bar{z}, \bar{x}, d(t)) .
\end{aligned}
$$

From (7) and (14), it is clear that $\phi_{0}(0,0, d)=0$.

On one hand, system (17) can be rewritten as

$$
\dot{\tilde{\eta}}=Q(\tilde{\eta}, \bar{z}, \bar{x}, d(t))=M \tilde{\eta}-N\left(\beta^{[2]}(\tilde{\eta}+\delta)-\beta^{[2]}(\delta)\right)+\psi(\bar{z}, \bar{x}, d(t))
$$

where $\delta=N \bar{x}_{r}+\theta$, and

$$
\begin{aligned}
\psi(\bar{z}, \bar{x}, d(t)) & =M N \bar{x}_{r}-N f_{1}\left(\bar{z}+\mathbf{z}(v, w), \bar{x}_{1}+\mathbf{x}_{1}(v, w), \cdots, \bar{x}_{r}+\mathbf{x}_{r}(v, w), v, w\right) \\
& +N f_{1}\left(\mathbf{z}(v, w), \mathbf{x}_{1}(v, w), \cdots, \mathbf{x}_{r}(v, w), v, w\right)-N\left(\beta^{[2]}\left(N \bar{x}_{r}+\theta\right)-\beta^{[2]}(\theta)\right) .
\end{aligned}
$$

Under assumption A4, the derivative of $V_{2}(\tilde{\eta})$ along system (17) is

$$
\begin{aligned}
\frac{d V_{2}(\tilde{\eta})}{d t} & =-\tilde{\eta}^{T} \tilde{\eta}-2 \tilde{\eta}^{T} P_{M} N\left(\beta^{[2]}(\tilde{\eta}+\delta)-\beta^{[2]}(\delta)\right)+2 \tilde{\eta} P_{M} \psi(\bar{z}, \bar{x}, d(t)) \\
& \leq-R \tilde{\eta}^{T} \tilde{\eta}+2 \tilde{\eta} P_{M} \psi(\bar{z}, \bar{x}, d(t)) \leq-\frac{R}{2}\|\tilde{\eta}\|^{2}+\frac{2}{R}\left\|P_{M} \psi(\bar{z}, \bar{x}, d(t))\right\|^{2} .
\end{aligned}
$$

Noting that $\psi(0,0, d(t))=0$, it is not difficult to find $C^{1}$ class $K_{\infty}$ functions $\sigma_{\bar{z}}$ and $\sigma_{\bar{x}}$, independent of $d(t)$, such that $\sigma_{\bar{z}}(\|\bar{z}\|) \leq\|\bar{z}\|^{2} \hat{\sigma}_{\bar{z}}(\|\bar{z}\|)$ for some smooth positive function $\hat{\sigma}_{\bar{z}}$, and

$$
\frac{d V_{2}(\tilde{\eta})}{d t} \leq-\frac{R}{2}\|\tilde{\eta}\|^{2}+\sigma_{\bar{z}}(\|\bar{z}\|)+\sigma_{\bar{x}}(\|\bar{x}\|) .
$$

On the other hand, under A3, by using the changing supply function technique [16], for any positive function $\Delta(\bar{z})$, there exist a smooth function $V_{1}^{\prime}(\bar{z})=$ $\int_{0}^{V_{1}(\bar{z})} S(s) d s$, where $S(s) \geq 1$ is a $C^{1}$ nondecreasing function, and class $K_{\infty}$ functions $\bar{\alpha}_{i}^{\prime}, i=1,2$, and $\sigma^{\prime}$, independent of $d(t)$, such that

$$
\begin{array}{r}
\bar{\alpha}_{1}^{\prime}(\|\bar{z}\|) \leq V_{1}^{\prime}(\bar{z}) \leq \bar{\alpha}_{2}^{\prime}(\|\bar{z}\|) \\
\frac{\partial V_{1}^{\prime}(\bar{z})}{\partial \bar{z}} \bar{f}_{0}(\bar{z}, \bar{x}, d(t)) \leq-\Delta(\bar{z}) \bar{\alpha}_{3}(\|\bar{z}\|)+\sigma^{\prime}(\|\bar{x}\|) .
\end{array}
$$

Since the function $\bar{\alpha}_{3}(\cdot)$ satisfies $\lim _{s \rightarrow 0^{+}} \sup \frac{\bar{\alpha}_{3}^{-1}\left(s^{2}\right)}{s}<+\infty$, we can choose $\Delta$ such that

$$
\Delta(\bar{z}) \bar{\alpha}_{3}(\|\bar{z}\|) \geq\|\bar{z}\|^{2}\left[\hat{\sigma}_{\bar{z}}(\|\bar{z}\|)+1\right] .
$$


Hence, we have $\Delta(\bar{z}) \bar{\alpha}_{3}(\|\bar{z}\|) \geq \sigma_{\bar{z}}(\|\bar{z}\|)+\|\bar{z}\|^{2}$. To show (19), note that for any smooth function $\bar{\Delta}(\bar{z})$, there exists a smooth function $\Delta(\bar{z})$ such that

$$
\Delta(\bar{z}) \bar{\alpha}_{3}(\|\bar{z}\|) \geq\|\bar{z}\|^{2} \bar{\Delta}(\bar{z}) .
$$

In fact, since $\bar{\alpha}_{3}(\cdot)$ satisfies $\lim _{s \rightarrow 0^{+}} \sup \frac{\bar{\alpha}_{3}^{-1}\left(s^{2}\right)}{s}<+\infty$, there exits some constant $L_{1} \geq 1$ such that $\frac{\|\bar{z}\|^{2}}{L_{1}^{2}} \leq \bar{\alpha}_{3}(\|\bar{z}\|)$ for $\|\bar{z}\| \leq 1$, and since $\bar{\alpha}_{3}(\cdot)$ is of class $K_{\infty}$, for some constant $L_{2}>0, \bar{\alpha}_{3}(\|\bar{z}\|) \geq L_{2}$ when $\|\bar{z}\| \geq 1$. As a result, (20) holds for

$$
\Delta(\bar{z}) \geq L_{1}^{2} \bar{\Delta}(\bar{z})+\frac{1}{L_{2}}\|\bar{z}\|^{2} \bar{\Delta}(\bar{z}) .
$$

Then, (19) holds from (20) by taking $\bar{\Delta}(\bar{z}) \geq \hat{\sigma}_{\bar{z}}(\|\bar{z}\|)+1$.

Now, let $W_{1}(\tilde{z})=V_{1}^{\prime}(\bar{z})+V_{2}(\tilde{\eta})$, then clearly, $\alpha_{1}(\|\tilde{z}\|) \leq W_{1}(\tilde{z}) \leq \alpha_{2}(\|\tilde{z}\|)$ for some class $K_{\infty}$ functions $\alpha_{i}, i=1,2$, and

$$
\begin{aligned}
& \frac{\partial W_{1}(\tilde{z})}{\partial \tilde{z}} \phi_{0}(\tilde{z}, \bar{x}, d(t)) \\
\leq & -\frac{R}{2}\|\tilde{\eta}\|^{2}+\sigma_{\bar{z}}(\|\bar{z}\|)+\sigma_{\bar{x}}(\|\bar{x}\|)-\Delta(\bar{z}) \bar{\alpha}_{3}(\|\bar{z}\|)+\sigma^{\prime}(\|\bar{x}\|) \\
\leq & -\frac{R}{2}\|\tilde{\eta}\|^{2}-\|\bar{z}\|^{2}+\sigma_{\bar{x}}(\|\bar{x}\|)+\sigma^{\prime}(\|\bar{x}\|) .
\end{aligned}
$$

Clearly, there exist class $K_{\infty}$ functions $\gamma_{1}$ and $\alpha_{3}$ such that

$$
\frac{\partial W_{1}(\tilde{z})}{\partial \tilde{z}} \phi_{0}(\tilde{z}, \bar{x}, d(t)) \leq-\alpha_{3}(\|\tilde{z}\|), \forall\|\tilde{z}\| \geq \gamma_{1}(\|\bar{x}\|) .
$$

Verification of Condition 2: Under assumption A5, it suffices to prove that $(\tilde{z}, \bar{x}) \in$ $\mathbb{O}_{c_{1}}^{1} \times \mathbb{O}_{c_{2}}^{2}$ implies $(\tilde{z}, \bar{x}) \in \mathbb{O}_{c_{1}}^{3} \times \mathbb{O}_{c_{2}}^{2}$. In fact, when $\tilde{z} \in \mathbb{O}_{c_{1}}^{1}$, we have

$$
W_{1}(\tilde{z})=V_{1}^{\prime}(\bar{z})+V_{2}(\tilde{\eta})=\int_{0}^{V_{1}(\bar{z})} S(s) d s+V_{2}(\tilde{\eta}) \leq c_{1},
$$

which gives

$$
\int_{0}^{V_{1}(\bar{z})} S(s) d s \leq c_{1} \text { and } V_{2}(\tilde{\eta}) \leq c_{1}
$$

Since $S(s) \geq 1$, we further get that $V_{1}(\bar{z}) \leq c_{1}$. As a result, $\tilde{z} \in \mathbb{O}_{c_{1}}^{3}$.

Verification of Condition 3: From inequality (21), it can be seen that $\alpha_{3}$ can be chosen such that locally, $\alpha_{3}(\|\tilde{z}\|) \geq \lambda_{1}\|\tilde{z}\|^{2}$ for some $\lambda_{1}>0$. Hence,

$$
\frac{\partial W_{1}(\tilde{z})}{\partial \tilde{z}} \phi_{0}(\tilde{z}, 0, d(t)) \leq \alpha_{3}(\|\tilde{z}\|) \leq-\lambda_{1}\|\tilde{z}\|^{2} .
$$

Second, since $\phi_{1}(\tilde{z}, 0, d)$ is $C^{1}$ and $d \in \mathbb{D}$, we have, locally, $\left|\phi_{1}(\tilde{z}, 0, d)\right| \leq \lambda_{2}\|\tilde{z}\|$ for some $\lambda_{2}>0$. 
Third, we have

$$
\begin{aligned}
& \frac{\partial W_{1}(\tilde{z})}{\partial \tilde{z}}\left[\phi_{0}(\tilde{z}, \bar{x}, d(t))-\phi_{0}(\tilde{z}, 0, d(t))\right] \\
\leq & {\left[S\left(V_{1}(\bar{z})\right) \frac{\partial V_{1}(\bar{z})}{\partial \tilde{z}}+2 P_{M} \tilde{\eta}\right]\left[\phi_{0}(\tilde{z}, \bar{x}, d(t))-\phi_{0}(\tilde{z}, 0, d(t))\right] . }
\end{aligned}
$$

Since $\phi_{0}(\tilde{z}, \bar{x}, d(t))$ is $C^{1}$, then under A3, we have, locally,

$$
\frac{\partial W_{1}(\tilde{z})}{\partial \tilde{z}}\left[\phi_{0}(\tilde{z}, \bar{x}, d(t))-\phi_{0}(\tilde{z}, 0, d(t))\right] \leq \lambda_{3}\|\tilde{z}\|\|\bar{x}\|
$$

for some $\lambda_{3}>0$. From above, Condition 3 holds with $\tilde{W}_{1}(\tilde{z})=W_{1}(\tilde{z})$.

REMARK 3.2. Roughly, assumption A3 implies that the subsystem $\dot{\bar{z}}=\bar{f}_{0}(\bar{z}$, $\bar{x}, d)$ is robust input-to-state stable with $\bar{x}$ as input, $\bar{z}$ as state and $d$ as uncertainty, and it also implies that the equilibrium point of $\dot{\bar{z}}=\bar{f}_{0}(\bar{z}, 0, d)$ at $\bar{z}=0$ is locally exponentially stable if the functions $\bar{\alpha}_{1}$ and $\bar{\alpha}_{2}$ take quadratic form in some neighborhood of the origin. A similar assumption can be found in [10] which handles the global robust regulation problem of lower triangular systems. This assumption together with assumption A4 guarantees that the zero dynamics of the augmented system satisfies Conditions 1 and 3. Assumption A5 is made to guarantee that the zero dynamics of the augmented system satisfies Conditions 2 .

REMARK 3.3. As pointed out in [3], assumption A4 is satisfied when $\mid \beta^{[2]}(\tilde{\eta}+\delta)$ $-\beta^{[2]}(\delta) \mid \leq \frac{(1-R)}{2\left\|P_{M} N\right\|}\|\tilde{\eta}\|$. In particular, assumption A4 is satisfied when $\beta(x)$ is a linear function of $x$.

By Proposition 2.1 and Theorem 3.1, we have the following conclusion.

THEOREM 3.2. Under assumptions A1-A5, the semi-global robust output regulation problem of the system (1) is solvable by a dynamic output feedback controller.

REMARK 3.4. The methodology of this paper is different from the one in [12]. In [12], the dynamics of the exosystem is treated as part of the augmented system. In this paper, the augmented system only consists of the given plant and the internal model while the exogenous signal $v$ is treated as a time-varying disturbance, which can be handled in the same way as the unknown parameter $w$. Therefore, the augmented system is still in the standard normal form. As a result, the semi-global robust stabilization technique in [13] can be directly applied to handle the robust stabilization problem of the augmented system.

4. An Example. Consider the following system

$$
\begin{aligned}
\dot{z} & =-z-z^{3}+w_{1} z\left(x_{1}-v_{1}\right)+x_{2}+v_{2} \\
\dot{x}_{1} & =x_{2} \\
\dot{x}_{2} & =w_{1} z-x_{1}+0.1 \sin ^{2}\left(w_{2} x_{1} x_{2}\right)+w_{2} v_{1} x_{2}+u \\
y & =x_{1} \\
e & =y-v_{1}
\end{aligned}
$$


with the exosystem

$$
\dot{v}_{1}=-v_{2}, \quad \dot{v}_{2}=v_{1} .
$$

It can be verified that the solution of the regulator equations associated with the system (22)-(23) is given by

$$
\begin{aligned}
& \mathbf{z}(v, w)=0, \quad \mathbf{x}_{1}(v, w)=v_{1}, \quad \mathbf{x}_{2}(v, w)=-v_{2} \\
& \mathbf{u}(v, w)=w_{2} v_{1} v_{2}-0.1 \sin ^{2}\left(w_{2} v_{1} v_{2}\right) .
\end{aligned}
$$

Thus, system (22)-(23) satisfies assumption A1. Since, $\mathbf{u}(v, w)$ is not a polynomial in $v$, the semi-global robust output regulation of this system cannot be solved by any existing approaches.

$$
\text { Let } \tau(v, w)=\left[\begin{array}{c}
\tau_{1}(v, w) \\
\tau_{2}(v, w)
\end{array}\right]=\left[\begin{array}{c}
w_{2} v_{1} v_{2} \\
w_{2}\left(v_{1}^{2}-v_{2}^{2}\right)
\end{array}\right] \text {. Then, assumption A2 is satisfied }
$$
with $\Gamma(\tau)=\tau_{1}-0.1 \sin ^{2}\left(\tau_{1}\right), \Phi=\left[\begin{array}{cc}0 & 1 \\ -4 & 0\end{array}\right]$, and $\Psi=\left[\begin{array}{ll}1 & 0\end{array}\right]$. Let $M=\left[\begin{array}{cc}-1 & 0 \\ 0 & -2\end{array}\right]$, and $N=\left[\begin{array}{l}1 \\ 2\end{array}\right]$. Solving the Sylvester equation $T \Phi-M T=N \Psi$ gives a nonsingular matrix $T=\left[\begin{array}{cc}0.2 & -0.2 \\ 0.5 & -0.25\end{array}\right]$. Thus,

$$
\begin{aligned}
\theta & =T \tau \\
\beta(\eta) & =\Gamma\left(T^{-1} \eta\right)=\left[\begin{array}{ll}
-5 & 4
\end{array}\right] \eta-0.1 \sin ^{2}\left(\left[\begin{array}{ll}
-5 & 4
\end{array}\right] \eta\right) \\
\beta^{[2]}(\eta) & =-0.1 \sin ^{2}\left(\left[\begin{array}{ll}
-5 & 4
\end{array}\right] \eta\right) .
\end{aligned}
$$

An internal model is given as follows

$$
\dot{\eta}=M \eta+N\left(u-\beta^{[2]}(\eta)\right) .
$$

Performing the coordinate and input transformation (5) converts the augmented system (22) and (25) into the following form

$$
\begin{aligned}
\dot{\tilde{\eta}}= & M \tilde{\eta}-N\left(\beta^{[2]}(\tilde{\eta}+\delta)-\beta^{[2]}(\delta)\right)+\psi(\bar{z}, \bar{x}, d) \\
\dot{\bar{z}}= & -\bar{z}-\bar{z}^{3}+w_{1} \bar{z} \bar{x}_{1} \\
\dot{\bar{x}}_{1}= & \bar{x}_{2} \\
\dot{\bar{x}}_{2}= & \beta\left(\tilde{\eta}+N \bar{x}_{2}+\theta\right)+w_{1} \bar{z}-\bar{x}_{1}+w_{2} v_{1}\left(\bar{x}_{2}-v_{2}\right) \\
& +0.1 \sin ^{2}\left(w_{2}\left(\bar{x}_{1}+v_{1}\right)\left(\bar{x}_{2}-v_{2}\right)\right)+\bar{u}
\end{aligned}
$$

where $\delta=N \bar{x}_{2}+\theta$, and

$$
\begin{aligned}
\psi(\bar{z}, \bar{x}, d)= & M N \bar{x}_{2}-N\left(\beta^{[2]}\left(N \bar{x}_{2}+\theta\right)-\beta^{[2]}(\theta)\right)-N\left(w_{1} \bar{z}+\bar{x}_{1}+w_{2} v_{1} \bar{x}_{2}\right. \\
& \left.-0.1 \sin ^{2}\left(w_{2} v_{1} v_{2}\right)\right)+0.1 \sin ^{2}\left(w_{2}\left(\bar{x}_{1}+v_{1}\right)\left(\bar{x}_{2}-v_{2}\right)\right)
\end{aligned}
$$


It can be verified that the system (26) satisfies the assumptions A3-A5, and then satisfies Condition 1-3. To be more specific, assume $\mathbb{V}=\left\{v_{1}^{2}+v_{2}^{2} \leq 1\right\}$, and $\mathbb{W}=$ $\left\{-1 \leq w_{i} \leq 1, \quad i=1,2\right\}, \mathbb{X}=\left\{\left|x_{i}\right| \leq 2, \quad i=1,2\right\}$ and $\mathbb{Z}=\{|z| \leq 1\}$. Let $V_{1}(\bar{z})=\frac{1}{2} \bar{z}^{2}$, then

$$
\frac{\partial V_{1}(\bar{z})}{\partial \bar{z}} \bar{f}_{0}(\bar{z}, \bar{x}, d)=-\bar{z}^{2}-\bar{z}^{4}+w_{1} \bar{z}^{2} \bar{x}_{1} \leq-\bar{z}^{2}+\frac{1}{4} \bar{x}_{1}^{2} \leq-\bar{z}^{2}+\frac{1}{4}\|\bar{x}\|^{2}
$$

that is, assumption A3 is satisfied with $\bar{\alpha}_{1}(s)=\bar{\alpha}_{2}(s)=\frac{1}{2} s^{2}, \bar{\alpha}_{3}(s)=s^{2}$ and $\sigma(s)=$ $\frac{1}{4} s^{2}$. Solving the Lyapunov equation $P_{M} M+M^{T} P_{M}=-I$ gives $P_{M}=\left[\begin{array}{ll}0.5 & 0 \\ 0 & 0.25\end{array}\right]$ then,

$$
\begin{aligned}
& \left.-2 \tilde{\eta}^{T}\left[\begin{array}{ll}
0.5 & 0 \\
0 & 0.25
\end{array}\right]\left[\begin{array}{l}
1 \\
2
\end{array}\right](\tilde{\eta}+\delta)-\beta^{[2]}(\delta)\right) \\
\leq & 0.1\left|\left(\tilde{\eta}_{1}+\tilde{\eta}_{2}\right)\left(-5 \tilde{\eta}_{1}+4 \tilde{\eta}_{2}\right)\right| \leq 0.6\|\tilde{\eta}\|^{2}
\end{aligned}
$$

that is, assumption A4 is satisfied with $0<R<0.4$. Now let $R=0.2$, and $V_{2}(\tilde{\eta})=$ $\tilde{\eta}^{T} P_{M} \tilde{\eta}$, then

$$
\begin{aligned}
\frac{d V_{2}(\tilde{\eta})}{d t} & \leq-0.1 \tilde{\eta}^{T} \tilde{\eta}+10\left\|P_{M} \psi(\bar{z}, \bar{x}, d)\right\|^{2} \\
& \leq-0.1 \tilde{\eta}^{T} \tilde{\eta}+\sigma_{\bar{z}}(\|\bar{z}\|)+\sigma_{\bar{x}}(\|\bar{x}\|)
\end{aligned}
$$

for $\sigma_{\bar{z}}(s)=20 s^{2}$ and $\sigma_{\bar{x}}(s)=173.2 s^{2}+0.01 s^{4}$. Let $W_{1}(\tilde{z})=20.1 V_{1}(\bar{z})+V_{2}(\tilde{\eta})$, then

$$
\begin{aligned}
\frac{\partial W_{1}(\tilde{z})}{\partial \tilde{z}} \phi_{0}(\tilde{z}, \bar{x}, d) & \leq-0.1 \tilde{\eta}^{T} \tilde{\eta}-0.1 \bar{z}^{2}+178.45\|\bar{x}\|^{2}+0.01\|\bar{x}\|^{4} \\
& \leq-0.05\|\tilde{z}\|^{2}, \quad \forall\|\tilde{z}\| \geq \sqrt{20\left(178.45\|\bar{x}\|^{2}+0.01\|\bar{x}\|^{4}\right)}
\end{aligned}
$$

thus, Condition 1 is satisfied with

$$
\alpha_{3}(s)=0.05 s^{2} \text { and } \gamma_{1}(s)=\sqrt{20\left(178.45 s^{2}+0.01 s^{4}\right)} .
$$

Let $K=\left[\begin{array}{ll}-1 & -2\end{array}\right]$, then solving the Lyapunov equation $P(A+B K)+(A+B K) P=$ $-I$ gives $P=\left[\begin{array}{ll}1.5 & 0.5 \\ 0.5 & 0.5\end{array}\right]$. Let $c_{1}=400$ and $c_{2}=12$, assumption A5, and thus Condition 2 , is satisfied with $\rho(\bar{x})=20+5\left|\bar{x}_{2}\right|$. Also, it is not difficult to verify that Condition 3 holds with $\tilde{W}_{1}(\tilde{z})=W_{1}(\tilde{z})$. Thus, by the approach detailed in [13], a state feedback controller that solves the stabilization problem for the system (26) is given as follows,

$$
u=k(\bar{x})=-q(\bar{x}) q_{s}(\bar{x})
$$

where $q(\bar{x})=20+5\left|\bar{x}_{2}\right|$, and

$$
q_{s}(\bar{x})= \begin{cases}\frac{s}{|s|}, & q(\bar{x})|s|>\mu>0 \\ q(\bar{x}) \frac{s}{\mu}, & q(\bar{x})|s| \leq \mu\end{cases}
$$


with $s=2 B^{T} P \bar{x}$ and $\mu=10$. To implement the state feedback controller (27) as an output feedback controller, we use the following high gain observer,

$$
\begin{aligned}
& \dot{\hat{x}}_{1}=\hat{x}_{2}+5.5\left(e-\hat{x}_{1}\right) / \epsilon \\
& \dot{\hat{x}}_{2}=6\left(e-\hat{x}_{1}\right) / \epsilon^{2} .
\end{aligned}
$$

with $\epsilon=0.04$. Finally, an output feedback controller solves the output regulation problem for the system (22) is given as follows,

$$
\begin{aligned}
u & =\beta(\eta)+k(\hat{x}) \\
\dot{\hat{x}}_{1} & =\hat{x}_{2}+5.5\left(e-\hat{x}_{1}\right) / \epsilon \\
\dot{\hat{x}}_{2} & =6\left(e-\hat{x}_{1}\right) / \epsilon^{2} \\
\dot{\eta} & =M \eta+N\left(k(\hat{x})+\Psi T^{-1} \eta\right) .
\end{aligned}
$$

The performance of the controller is simulated for the case where $w_{1}=0.7$, $w_{2}=-0.5, z(0)=0.5, x(0)=\left[\begin{array}{ll}1.5 & 1\end{array}\right]^{T}, \eta(0)=0, \hat{x}(0)=0$ and is shown in Figure 1.

5. Conclusions. This paper addresses the semi-global robust output regulation problem for a class of minimum phase nonlinear systems by using dynamic output feedback. Under the framework of [3], the problem is solved in two steps. In the first step, the semi-global robust output regulation problem is converted into a semiglobal robust stabilization problem, and then in the second step, the semi-global robust output regulation problem is solved by solving the corresponding semi-global robust stabilization problem with min-max control proposed in [13]. Moreover, by introducing a nonlinear internal model, the polynomial condition on the solution of the regulator equations is relaxed.

\section{REFERENCES}

[1] C. I. Byrnes, F. Delli Priscoli, A. Isidori, And W. Kang, Structurally stable output regulation of nonlinear systems, Automatica, 33(1997), pp. 369-385.

[2] J. HuAng, Remarks on the robust output regulation problem for nonlinear systems, IEEE Transactions on Automatic Control, 46(2001), pp. 2028-2031.

[3] J. HuAng And Z. Chen, A general framework for tackling the output regulation problem, IEEE Transactions on Automatic Control, 49(2004), pp. 2203-2218.

[4] J. HuAng And C. F. Lin, On a robust nonlinear servomechanism problem, IEEE Transactions on Automatic Control, 39(1994), pp. 1510-1513.

[5] J. HuAng And W. J. Rugh, On a nonlinear multivariable servomechanism problem, Automatica, 26(1990), pp. 963-972.

[6] J. Huang And W. J. Rugh, Stabilization on zero-error manifolds and the nonlinear servomechanism problem, IEEE Transactions on Automatic Control, 37(1992), pp. 1009-1013.

[7] A. IsIDORI, A remark on the problem of semiglobal nonlinear output regulation, IEEE Transactions on Automatic Control, 42(1997), pp. 1734-1738.

[8] A. Isidori And C. I. Byrnes, Output regulation of nonlinear systems, IEEE Transactions on Automatic Control, 35(1990), pp. 131-140. 
[9] A. Isidori, L. MARConi, ANd A. Serrani, New results on semiglobal output regulation of nonminimum-phase nonlinear systems, in: Proc. 41st IEEE Conf. on Decision and Control, pp. 1467-1472, 2002.

[10] Z. P. Jiang And L. Praly, Design of robust adaptive controllers for nonlinear systems with dynamic uncertainties, Automatica, 34(1998), pp. 825-840.

[11] H. KHALIL, Robust servomechanism output feedback controllers for feedback linearizable systems, Automatica, 30(1994), pp. 1587-1589.

[12] H. KHALIL, On the robust servomechanisms for minimum phase nonlinear systems, International Journal of Robust Nonlinear Control, 10(2000), pp. 339-361.

[13] N. A. Mahmoud and H. K. Khalil, Asymptotic stabilization of minmum phase nonlinear systems using output feedback, Proceedings of the $32^{\text {th }}$ Conference on Decision and Control, pp. 1960-1965, San Antonie, Taxas, 1993.

[14] N. A. Mahmoud And H. K. Khalil, Asymptotic regulation of minimum phase nonlinear systems using output feedback, IEEE Transactions on Automatic Control, 41(1996), pp. 1402-1412.

[15] E. D. Sontag, Smooth stabilization implies coprime factorization, IEEE Transaction on Automatic Control, 34(1989), pp. 435-443.

[16] E. Sontag And A. TeEL, Changing supply function in input/state stable systems, IEEE Transactions on Automatic Control, 40(1995), pp. 1476-1478.

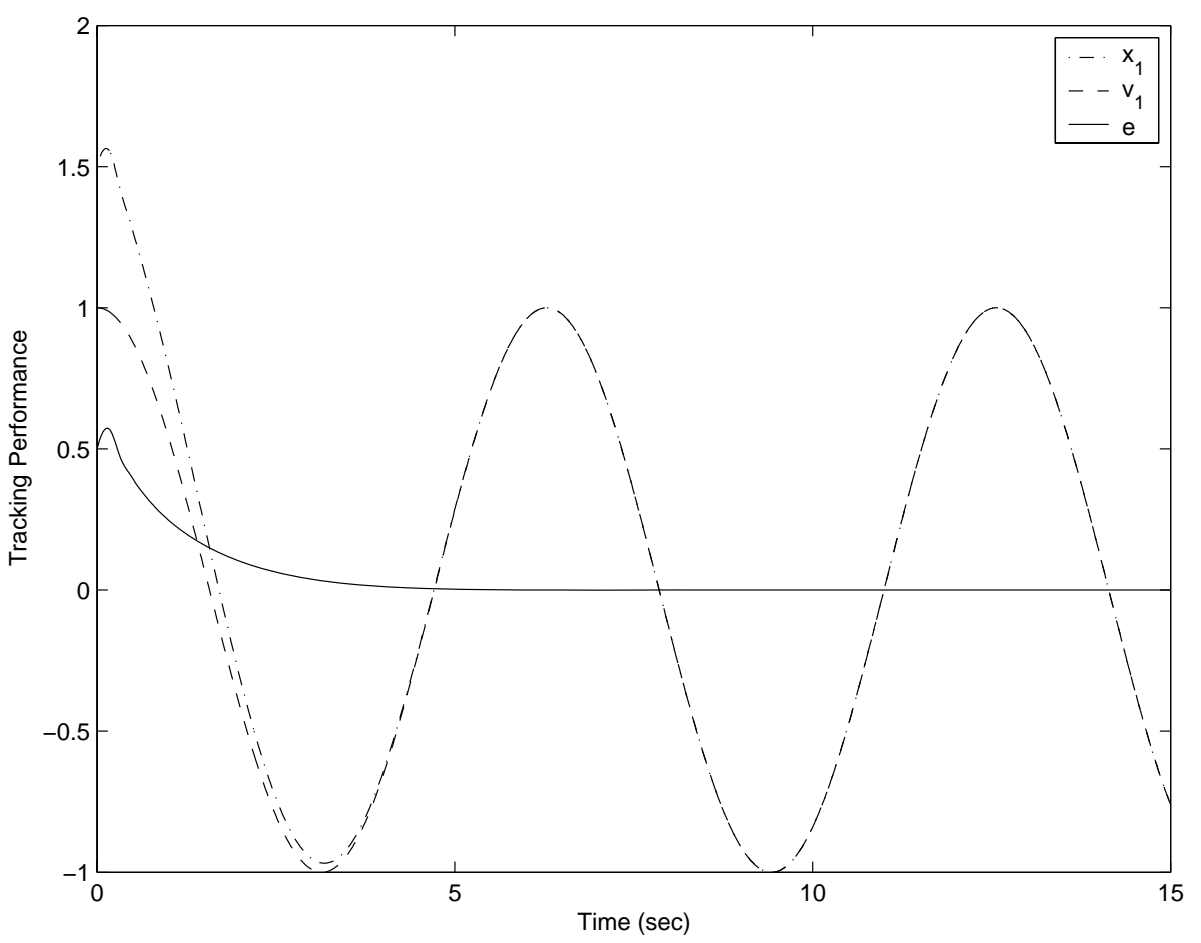

FIG. 1. Tracking performance of the closed-loop system 